\title{
TAMANHO DA SEMENTE E SUBSTRATOS NA GERMINAÇÃO E CRESCIMENTO INICIAL DE MUDAS DE CAGAITEIRA ${ }^{1}$
}

\author{
Seeds sizes and substrates on germination of seed and initial grownth of cagaiteira
}

\author{
Silvia Nietsche ${ }^{2}$, Valdeir Dias Gonçalves ${ }^{3}$, Marlon Cristian Toledo Pereira ${ }^{2}$, \\ Fernando Almeida Santos ${ }^{3}$, Samuel Campos de Abreu ${ }^{3}$, Wagner Ferreira da Mota $^{2}$
}

\section{RESUMO}

A cultura da cagaiteira apresenta perspectivas de expansão no cerrado e no Nordeste brasileiro. Com o presente trabalho tevese como objetivo avaliar as características de germinação de sementes de cagaiteira de diferentes tamanhos em três tipos de substratos. O delineamento experimental utilizado foi de blocos inteiramente casualizados em um esquema de parcela subdividida, com fatorial (2 x3), consistindo de dois tamanhos de sementes, grandes (13,5 mm de largura, 15,91 mm de comprimento e 1,41 g de massa) e pequenas (9,94 mm de largura, 12,19 mm de comprimento e 0,68 g de massa) em três tipos de substratos (plugmix; areia, argila e esterco de curral, na proporção v/v 1:1:1; e areia e argila na proporção v/v 1:1). Pelos resultados, observou-se que, independentemente do tamanho da semente e do tipo de substrato, não houve diferenças significativas quanto à porcentagem de emergência, que foi superior a 90\%. Nas características de índice de velocidade de emergência, altura e número de folhas, observou-se superioridade para as plantas provenientes de sementes grandes. Em relação aos diferentes tipos de substratos, houve diferenças significativas para as características de altura e número de folhas, indicando a superioridade dos substratos à base de areia e argila.

Termos para indexação: Eugenia dysenterica, emergência, propagação.

\begin{abstract}
The culture of cagaita presents perspectives of expansion in the open pasture and Brazilian northeast, this culture is usually spread by seeds. This work aimed at evaluating the characteristics of germination of different sizes of seeds on three types of substrates. The experimental was designed in blocks at random outlined in subparcels with four replications, in a factorial system of two seed sizes, big and small, on three types of substrates (plugmix, sand; clay and manure; sand and clay). The results showed that regardless of the seed size there were not significant differences for germination percentage. When analyzed characteristic of germination speed index, height and leaf number, significant superiority was observed for the plants from big seeds. Significant differences for characteristics of height and leaf number were also observed indicating superiority of sand and clay substratum. We can conclude that independently of the substratum type, percentages of cagaita seed germination are over $90 \%$, but plantlets initial growth may be damaged if the nurseryman does not use a balanced and appropriate substratum to the specie.
\end{abstract}

Index terms: Eugenia dysenterica, emergency, propagation.

(Recebido para publicação em 13 de janeiro de 2004 e aprovado em 30 de novembro de 2004)

\section{INTRODUÇÃO}

A cagaiteira (Eugenia dysenterica DC.) é uma árvore do cerrado que pode alcançar até $10 \mathrm{~m}$ de altura; a casca do tronco é profundamente sulcada e os ramos são tortuosos, característicos de espécies do cerrado. As folhas são opostas, ovais e elípticas, com consistência cartácea e ausente na floração. Apresenta inflorescência ramificada com até nove flores brancas e os frutos são carnosos, de cor amarela na maturidade, com 1 a 4 sementes (SILVA, 1998).

Os teores de vitamina C da cagaita $(18,28$ $\mathrm{mg} / 100 \mathrm{~g})$ são superiores aos encontrados na banana d'água madura e na maçã-argentina, que são de 6,4 e $5,9 \mathrm{mg} / 100 \mathrm{~g}$, respectivamente. Em relação aos ácidos graxos poliinsaturados, a cagaita apresenta maior teor do ácido linoléico, em comparação aos encontrados em oliva, dendê e coco, e o teor de ácido linolênico é superior aos encontrados em milho, girassol, amendoim, soja, oliva e dendê (FRANCO, 1992; SANO e ALMEIDA, 1998).

De acordo com Simão (1998), as sementes de muitas espécies frutíferas, principalmente daquelas que possuem frutos carnosos, germinam tão logo sejam colocadas em condições de solo e ambientes favoráveis; porém, outras, nas mesmas condições, não germinam. Essa dormência pode ser causada por um impedimento físico, que impede a embebição da semente e a oxigenação do embrião, que permanece latente (LEMOS et al., 1987).

1. Monografia de conclusão do Curso de Agronomia da Universidade Estadual de Montes Claros, Caixa Postal 91 - $39440-000$ - Janaúba, MG.

2. Engenheiros Agrônomos, Dr., Sc. Professores do Departamento de Ciências Agrárias/UNIMONTES.

3. Estudantes do Curso de Agronomia/UNIMONTES. 
No Brasil, especialmente no Nordeste e Centro-Oeste, verifica-se uma enorme carência de oferta de mudas produzidas com tecnologia que garanta a qualidade genética e fitossanitária e, conseqüentemente, assegure o fortalecimento econômico da exploração e atenda às exigências dos mercados consumidores. As exigências dos consumidores têm aumentado cada vez mais no que se refere aos aspectos de sanidade das mudas e também quanto à qualidade e conformidade dos produtos ofertados, além da preocupação ambiental (OLIVEIRA et al., 2002). Assim, na produção de mudas de cagaiteira, a utilização de substratos alternativos, a procura de recipientes e adubações mais adequadas a essa atividade têm sido objetivo de pesquisas visando à diminuição de custos sem, no entanto, perder de vista a qualidade do produto final.

De acordo com recomendações de Silva (1999), as sementes de cagaita devem ser semeadas a dois centímetros de profundidade e a germinação é de 95\% em um intervalo de quarenta a sessenta dias, índice considerado alto para fruteiras do cerrado. Sua propagação é feita quase que exclusivamente via semente, gerando grande variabilidade de plantas e frutos. Também é possível propagar a cagaiteira por enraizamento de estacas com aplicação de reguladores de crescimento, com até $90 \%$ de enraizamento após 180 dias, possibilitando selecionar genótipos superiores e propagar vegetativamente (PEREIRA et al., 2002).

Objetivou-se com o presente trabalho avaliar as características de emergência de sementes de cagaiteira de diferentes tamanhos em três tipos de substratos.

\section{MATERIAL E MÉTODOS}

Frutos maduros de cagaiteira foram coletados na Fazenda Recanto dos Araçás, em uma área de cerrado no município de Montes Claros, região norte de Minas Gerais, no dia 10/11/2002. Os frutos foram despolpados manualmente e as sementes foram lavadas em água e colocadas para secar à sombra, sendo classificadas em dois tamanhos: grandes $(13,5 \mathrm{~mm}$ de largura, 15,91 mm de comprimento e 1,41 g de massa) e pequenas $(9,94 \mathrm{~mm}$ de largura, $12,19 \mathrm{~mm}$ de comprimento e 0,68 g de massa), descartando aquelas que apresentassem alguma injúria mecânica ou algum tipo de problema fitossanitário. O delineamento experimental utilizado foi de blocos inteiramente casualizados em um esquema de parcela subdividida no tempo, tendo nas parcelas um fatorial ( $2 \times 3)$, sendo dois tamanhos de sementes e três tipos de substratos, e nas subparcelas, as épocas de avaliação, com quatro repetições e quatro sacos por parcela. Sementes grandes e pequenas de cagaiteira foram plantadas em sacos de polietileno $(15 \mathrm{~cm} \mathrm{x} 30 \mathrm{~cm})$ contendo os substratos: Sb - Substrato comercial, Plugmix; AT- Areia e terra de barranco na proporção v/v 1:1:1; e ATE - Areia, terra de barranco e esterco curtido na proporção $\mathrm{v} / \mathrm{v} 1: 1$. As sementes foram semeadas no dia 14/11/2002. A emergência foi controlada diariamente, até 75 dias após a semeadura, para fins de cálculo do índice de velocidade de emergência, sendo utilizada a formula: IVE = E1/N1 + E2/N2 ... + En/Nn; em que IVE = índice de velocidade de emergência; E1, E2 ... En = Números de plântulas normais emergidas na primeira, segunda até a última contagem; N1,N2 ... Nn = Número de dias da semeadura à primeira, segunda até a última contagem (NAKAGAWA, 1994). Foram avaliados também altura de plantas e número de folhas a cada quinze dias após a emergência.

As características avaliadas foram submetidas à análise de variância, com desdobramentos das interações significativas. Para as características de número de folhas e altura, foram testadas e ajustadas as equações de regressão, sendo o índice de velocidade de emergência comparado pelo teste de Tukey a 5\% de probabilidade.

\section{RESULTADOS E DISCUSSÃO}

Observou-se aumento linear da altura de plantas com o aumento dos dias após a semeadura, demonstrando adaptação aos três substratos testados (Figura 1). A maior média de altura foi constatada aos 182 dias, após a semeadura, com média de $6,00 \mathrm{~cm}$. No período compreendido entre 77 e 182 dias as plântulas cresceram em média $0,20 \mathrm{~cm}$ a cada 15 dias. O crescimento lento das mudas está associado à própria espécie (SOUZA et al., 2002), bem como pode ter sido também em função da ausência de nutrientes no substrato, visto que não foram efetuadas adubações de cobertura durante o experimento. Silva (1999) obteve mudas de cagaiteira com altura e diâmetro médios, aos 250 dias após a semeadura, de 5,56 $\mathrm{cm}$ e 0,14 cm, respectivamente. Segundo Chaves (2000), a escolha do substrato deve ser realizada em função da disponibilidade de materiais, de suas características físicas e químicas, de seu peso e custo, além da sua formulação. 
A maior média de número de folhas foi obtida aos 122 dias após a semeadura, com 3,4 folhas emitidas; após esse período, foi observado um decréscimo na emissão de novas folhas, alcançando média de 3,0 folhas aos 182 dias após o plantio (Figura 2). Esse decréscimo na emissão de novas folhas a partir dos 122 dias pode estar associado à baixa disponibilidade de nutrientes nos substratos testados, visto que foi efetuada apenas a adubação fosfatada no momento do plantio (Figura 2). Na produção de mudas, a utilização de substratos alternativos, menos onerosos, e a procura de recipientes e adubações mais adequadas a essa atividade têm sido objetivo de muitas pesquisas, visando à diminuição de custos sem, no entanto, perder de vista a qualidade do produto final (OLIVEIRA et al., 2002).

Em relação aos tipos de substratos, foram detectadas diferenças para as características de número de folhas e altura de plantas (Tabela 1). Os melhores resultados foram obtidos quando as sementes foram plantadas no substrato tipo AT (areia:terra de barranco), na proporção de 1:1, com médias de 4,26 e 6,37 cm, respectivamente. Esses resultados são muito importantes para o pequeno produtor do norte de Minas, que apresenta características de baixo índice de adoção de novas tecnologias, bem como pouca disponibilidade de recursos para investimento na propriedade. De acordo com Menezes et al. (2002), na produção de mudas, vários aspectos tecnológicos têm recebido a atenção dos pesquisadores, especialmente no que diz respeito à formação perfeita da plântula e facilidade no transplante, embora ainda se tenha pouca muita informação sobre o tipo de substrato que propicie a melhor formação da muda e desenvolvimento inicial da planta. Com relação ao índice de velocidade de emergência (I.V.E) (Tabela 1), não foram detectadas diferenças em relação aos substratos avaliados. A melhor média foi obtida em substrato contendo terra, areia e esterco, na proporção 1:1:1 v/v, com 0,9 sementes emergidas por dia. Em estudos realizados com sementes de graviola, determinou-se um I.V.E de 0,91 semente/dia, em condições de escarificação em liquidificador por cinco segundos (LEDO e CABANELAS, 1997). Esse resultado e outros estudos demonstram que essa espécie apresenta germinação satisfatória, porém, com crescimento lento, bem como ampla adaptação a situações de baixa concentração de nutrientes e matéria orgânica (SANO e ALMEIDA, 1998; SOUZA et al., 2002).

FIGURA 1 - Altura de plantas de cagaiteira 77 dias após a emergência, Unimontes, Janaúba, MG, 2002.
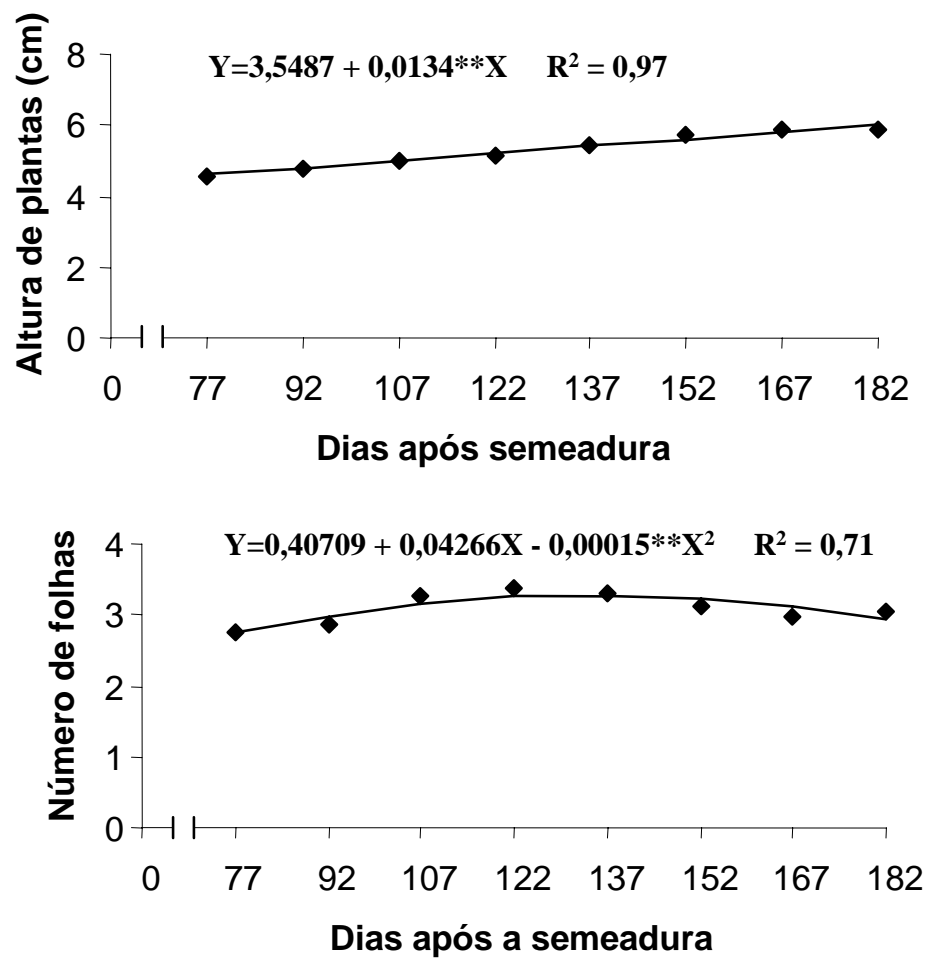

FIGURA 2 - Número de folhas em plantas de cagaiteira 77 dias após a emergência, Unimontes, Janaúba, MG, 2002. 
Em relação ao tamanho das sementes, pode-se destacar que houve diferenças significativas para as três características. Sementes maiores apresentaram superioridade no IVE (Índice de Velocidade de Emergência), número de folhas e altura de plantas, com médias de $0,10,3,37 \mathrm{~cm}$ e 5,85 cm, respectivamente (Tabela 2). Sementes de espécies de dicotiledôneas apresentam cotilédones suculentos e que ocupam o maior volume da semente, e durante o processo de germinação, todo o endosperma é absorvido pelo embrião, garantindo, dessa forma o crescimento inicial da plântula (RAVEN et al., 1992). Pelos resultados do presente trabalho, demonstra-se que ao selecionar sementes maiores de cagaiteira, essas apresentarão maiores quantidades de reservas nos seus cotilédones e, conseqüentemente, maior emergência e crescimento inicial das plântulas, garantindo maior possibilidade de sucesso na formação da muda.

Estudos realizados por Souza et al. (2002) confirmam a influência dos substratos no crescimento i- nicial e na sobrevivência de mudas de cagaiteira. Neste trabalho, o substrato mais adequado à produção das mudas consistiu de solo, terriço da mata e vermiculita, afirmando a grande adaptação dessa espécie a substratos com baixa e/ou nenhuma adubação química. A composição do substrato tem sido pesquisada intensamente, visando à obtenção de meios de crescimentos que apresentem composição uniforme, baixa densidade, alta capacidade de troca catiônica por unidade de peso seco, alta capacidade de retenção de água, boa aeração e drenagem, proporcionando condições ideais para o crescimento e desenvolvimento das mudas (Gomes et al., 1985).

A melhoria do sistema de produção de mudas de cagaiteira é necessária devido ao aumento na procura de espécies nativas para a produção comercial, bem como para reflorestar e recuperar áreas de cerrado degradado. Para tanto, estudos associados à propagação via semente são essenciais no auxílio à manutenção da variabilidade genética da espécie.

TABELA 1 - Número de folhas, altura de plantas e índice de velocidade de emergência (IVE) a partir da emergência de sementes de cagaiteira plantadas em três substratos, areia e terra (AT, 1:1 v/v), areia, terra e esterco (ATE, 1:1:1 v/v) e substrato comercial Plugmix (Sb), Unimontes, Janaúba, MG, 2002.

Tipos de Substratos Número de folhas

\begin{tabular}{|r} 
Tipos de Subs \\
\hline AT \\
ATE
\end{tabular}

$\mathrm{Sb}$
Índice de Velocidade de Emergência

Médias seguidas da mesma letra nas colunas não diferem entre si, a $5 \%$ de probabilidade, pelo teste de Tukey.

TABELA 2 - Número de folhas, altura de plantas e índice de velocidade de emergência (IVE) a partir da emergência de sementes grandes e pequenas de cagaiteira plantadas em três substratos diferentes, Unimontes, Janaúba, MG, 2002.

\begin{tabular}{cccc}
\hline Tamanho de Semente & Número de folhas & Altura de plantas (cm) & $\begin{array}{c}\text { Índice de Velocidade de } \\
\text { Emergência }\end{array}$ \\
\hline Pequenas & $2,79 \mathrm{~b}$ & $4,74 \mathrm{~b}$ & $0,08 \mathrm{~b}$ \\
Grandes & $3,37 \mathrm{a}$ & $5,85 \mathrm{a}$ & $0,10 \mathrm{a}$ \\
\hline
\end{tabular}

Médias seguidas da mesma letra nas colunas não diferem entre si, a $5 \%$ de probabilidade, pelo teste de Tukey. 


\section{CONCLUSÕES}

a) Melhores resultados foram obtidos com o substrato areia e terra;

b) Sementes maiores de cagaiteira propiciam melhor desenvolvimento inicial das plântulas.

\section{AGRADECIMENTOS}

À Fundação de Amparo à Pesquisa do Estado de Minas Gerais - FAPEMIG, pela concessão da bolsa, e ao Sr. Gualter Pereira de Oliveira, pelo apoio na realização deste trabalho.

\section{REFERÊNCIAS BIBLIOGRÁFICAS}

CHAVES, J. C. M. Normas de produção de mudas. Fortaleza: EMBRAPA Agroindústria Tropical, 2000. 37 p. (Documentos, 41).

FRANCO, G. Tabela de composição química dos alimentos. 9. ed. São Paulo: Atheneu, 1992. 307 p.

GOMES, J. M.; COUTO, L. P.; PEREIRA, A. R. Uso de diferentes substratos na produção de mudas de Eucalyptus grandis em tubetes e em bandejas de isopor. Revista Árvore, Viçosa, v. 89, n. 1, p. 58-86, 1985.

LEDO, A. S.; CABANELAS, C. I. L. Superação de dormência de sementes de graviola (Annona muricata L.). Revista Brasileira de Fruticultura, Cruz das Almas, v. 19, p. 397-400, 1997.

LEMOS, E. E. P. de et al. Germinação de sementes de pinha submetidas a tratamentos para quebra de dormência. In: CONGRESSO BRASILEIRO DE FRUTICULTURA, 9., 1987, Campinas, SP. Anais... Campinas: SBF/CBF, 1987. v. 2, p. 675-678.

MENEZES, L. S. et al. Efeito de substrato na produção de mudas de pinheira (Annona squamosa L.) em bandejas de isopor. In: CONGRESSO BRASILEIRO DE FRUTICULTURA, 17., 2002, Belém. Anais... Belém: SBF/CBF, 2002. CD-ROM.
NAKAGAWA, J. Testes de vigor baseados na avaliação de plântulas. In: VIEIRA, R. D.; CARVALHO, N. M. (Eds.). Teste de vigor em sementes. Jaboticabal: FUNEP, 1994. p. 49-85.

OLIVEIRA, E. M.; CORREIA, D.; CAVALCANTI JÚNIOR, A. T. Efeitos de substratos e fertilizante de liberação lenta na formação de porta-enxerto de cajueiro anão precoce (Anacardium occidentale L.) em tubetes. In: CONGRESSO BRASILEIRO DE FRUTICULTURA, 17., 2002, Belém. Anais... Belém: SBF/CBF, 2002. CD-ROM.

PEREIRA, E. B. C. et al. Avaliação preliminar do enraizamento de estacas de cagaiteira (Eugenia dysenterica D.C.). In: CONGRESSO BRASILEIRO DE FRUTICULTURA, 17., 2002, Belém. Anais... Belém: SBF/CBF, 2002. CD-ROM.

RAVEN, P. H.; EVERT, R. F.; EICHHORN, S. E. Biologia vegetal. 5. ed. Rio de Janeiro: Guanabara Koogan, 1992. $728 \mathrm{p}$.

SANO, S. M.; ALMEIDA, S. P. Cerrado: ambiente flora. Planaltina: EMBRAPA-CPAC, 1998. 556 p.

SILVA, S. R. Plantas do cerrado: utilizadas pelas comunidades da região do Grande Sertão Veredas. Brasília: Fundação Pró-natureza-FUNATURA, 1998. $109 \mathrm{p}$.

SILVA, R. S. M. Caracterização de subpopulações de cagaita (Eugenia dysenterica DC.) do sudeste de Goiás. 1999. 107 f. Dissertação (Mestrado em Agronomia) - Universidade Federal de Goiás, Goiânia, 1999.

SIMÃO, S. Tratado de fruticultura. Piracicaba: FEALQ, 1998. 760 p.

SOUZA, E. B. et al. Crescimento e sobrevivência de mudas de cagaiteira (Eugenia dysenterica DC) nas condições de cerrado. Revista Brasileira de Fruticultura, Jaboticabal, v. 24, n. 2, p. 491-495, 2002. 